\title{
In patients with metastatic breast cancer the identification of circulating tumor cells in epithelial-to-mesenchymal transition is associated with a poor prognosis
}

Michela Bulfoni ${ }^{1}$, Lorenzo Gerratana ${ }^{1,2}$, Fabio Del Ben ${ }^{1}$, Stefania Marzinotto ${ }^{3}$, Marisa Sorrentino ${ }^{1,3}$, Matteo Turetta ${ }^{1,3}$, Giacinto Scoles', Barbara Toffoletto', Miriam Isola', Carlo Alberto Beltrami', Carla Di Loreto ${ }^{1,3}$,

Antonio Paolo Beltrami ${ }^{*}$, Fabio Puglisi ${ }^{1,2}$ and Daniela Cesselli ${ }^{*}$

\begin{abstract}
Background: Although recent models suggest that the detection of Circulating Tumor Cells (CTC) in epithelialto-mesenchymal transition (EM CTC) might be related to disease progression in metastatic breast cancer (MBC) patients, current detection methods are not efficient in identifying this subpopulation of cells. Furthermore, the possible association of EM CTC with both clinicopathological features and prognosis of MBC patients has still to be demonstrated. Aims of this study were: first, to optimize a DEPArray-based protocol meant to identify, quantify and sort single, viable EM CTC and, subsequently, to test the association of EM CTC frequency with clinical data.

Methods: This prospective observational study enrolled $56 \mathrm{MBC}$ patients regardless of the line of treatment. Blood samples, depleted of CD45 ${ }^{\text {pos }}$ leukocytes, were stained with an antibody cocktail recognizing both epithelial and mesenchymal markers. Four $C D 45^{\text {neg }}$ cell subpopulations were identified: cells expressing only epithelial markers (E CTC), cells co-expressing epithelial and mesenchymal markers (EM CTC), cells expressing only mesenchymal markers (MES) and cells negative for every tested marker (NEG). CTC subpopulations were quantified as both absolute cell count and relative frequency. The association of CTC subpopulations with clinicopathological features, progression free survival (PFS), and overall survival (OS) was explored by Wilcoxon-Mann-Whitney test and Univariate Cox Regression Analysis, respectively.
\end{abstract}

Results: By employing the DEPArray-based strategy, we were able to assess the presence of cells pertaining to the above-described classes in every MBC patient. We observed a significant association between specific CD45 neg subpopulations and tumor subtypes (e.g. NEG and triple negative), proliferation (NEG and Ki67 expression) and sites of metastatic spread (e.g. E CTC and bone; NEG and brain). Importantly, the fraction of CD45 ${ }^{\text {neg }}$ cells co-expressing epithelial and mesenchymal markers (EM CTC) was significantly associated with poorer PFS and OS, computed, this latter, both from the diagnosis of a stage IV disease and from the initial CTC assessment.

Conclusion: This study suggests the importance of dissecting the heterogeneity of CTC in MBC. Precise characterization of CTC could help in estimating both metastatization pattern and outcome, driving clinical decision-making and surveillance strategies.

\footnotetext{
*Correspondence: antonio.beltrami@uniud.it; daniela.cesselli@uniud.it

MB and LG share the first authorship.

DC and FP share the last authorship.

'Department of Medical and Biological Sciences, University of Udine, P.le

Kolbe 4, 33100 Udine, Italy

Full list of author information is available at the end of the article
} 


\section{Background}

Circulating tumor cells (CTC) are rare cells shed into the bloodstream from primary tumors and metastases [1]. Since these latter represent the major cause of cancer-associated mortality [2], CTC isolation and characterization is one of the most active areas of translational cancer research [1]. In fact, CTC might represent an active source of metastatic spread from a primary tumor to secondary lesions [3, 4], and their role as a prognostic biomarker has been robustly demonstrated both in primary and metastatic cancer [5-9].

Moreover, detection and enumeration of CTC could serve as an early marker of response to systemic therapy, whereas the molecular characterization of CTC could lead to individualized targeted treatments, possibly sparing patients unnecessary and ineffective therapies [10].

Current models suggest that the invasive phenotype of breast cancers is mostly associated with an epithelial-tomesenchymal transition (EMT) [11]. This process leads to the expression of mesenchymal markers on tumor cells, which is paralleled by an increase in the migration and invasion properties of tumor cells, as well as in their resistance to apoptosis and ability to evade the immune response [11]. The detection of CTC that express either mesenchymal and epithelial mRNAs or only mesenchymal mRNAs could therefore be related, in metastatic breast cancer $(\mathrm{MBC})$ patients, to disease progression [12]. However, existing detection methods are not efficient in identifying CTC in EMT. In fact, the only Food and Drug Administration (FDA)-approved device to detect CTC, the CellSearch System (Veridex, Warren, NJ, USA), allows counting only epithelial cell adhesion molecule (EpCAM)-positive epithelial CTC. Moreover, this device does not allow harvesting viable CTC suitable for downstream analyses. For this reason, in the last years several innovative strategies to enrich, detect, count, and/or molecularly characterize CTC have been developed [13]. However, for most of these a clinical validation is still missing [14].

DEPArray (Silicon Biosystems, Bologna, Italy) is a dielectrophoresis-based platform able to handle a relatively small number of cells. The device is aimed at analyzing and sorting single, viable, rare cells thanks to an image-based selection process and to the entrapment of cells inside dielectrophoretic cages. Selected cells can be individually moved by software-controlled modulation of electrical fields and ultimately recovered for downstream molecular analyses [15].

The main objectives of our study were: to develop a novel strategy to enrich blood samples in CTC, independently from the expression of epithelial markers; to take advantage of the DEPArray system to identify and sort, based on a multiparametric fluorescence analysis, single, viable epithelial-like CTC as well as CTC in EMT; to explore, in a prospective observational case study including 56 patients with $\mathrm{MBC}$, the association between clinicopathological features, CTC number, and distribution of CTC subpopulations; and, finally, to provide evidence of the possible prognostic role of the enumeration of CTC in EMT.

\section{Methods \\ Patient population, ethics, consent, and permissions}

Our prospective observational study was approved by the Local Ethics Committee (decision No. 152/2011/ Sper) and, subsequently, by the Regional Ethics Committee (amendment No. 178/2014/Em). Fifty-six patients were enrolled by the Department of Oncology, University Hospital of Udine, Italy. Of these, 47 blood samples were analyzed for the presence of CTC by the Department of Pathology, University Hospital of Udine, Italy. Nine patients were excluded from the analysis because the volume of sampled blood was inadequate $(n=1)$, the timing of blood sampling was incorrect $(n=3)$, or the samples were employed to optimize the technical procedure $(n=5)$. All patients gave their written informed consent before their enrollment.

\section{Patient enrollment and follow-up}

The eligibility criteria for patient recruitment were as follows: female, adult ( $\geq 18$ years) patients with measurable MBC, at the start of a new systemic therapy, without limits to number and kind of previous therapies (hormone therapy, chemotherapy, targeted therapy), and Eastern Cooperative Oncology Group performance status (ECOG PS) score $\leq 2$. A histological sample representative of the primary tumor had to be available.

Before starting a new treatment, patients underwent baseline blood sampling for CTC evaluation (see later), and standard clinical studies.

Standard Response Evaluation Criteria In Solid Tumors (RECIST) criteria were used to determine patients' responses to treatment [16].

An expert pathologist reviewed the biopsies of every enrolled patient, defining the tumor type following the WHO classification of breast cancer [17], grading as in [18], and tumor subtype as in [19].

\section{Sampling of biologic material}

Blood samples of $15 \mathrm{ml}$ of blood were collected from 56 MBC patients and 27 healthy donors. Ethylenediamine tetraacetate was used as anticoagulant. The samples were processed within 4 hours from blood withdrawal. Healthy control blood samples were collected from female blood donors aged $20-60$ years. 


\section{Flow cytometry}

Epithelial-like (ER ${ }^{+} / \mathrm{PR}^{+} / \mathrm{HER} 2^{-}$MCF-7; ATCC, Teddington, Middlesex, UK) and mesenchymal-like (triple-negative MDA-MB231; ATCC, Teddington, Middlesex, UK) breast cancer cell lines were tested by flow cytometry, to determine their immunophenotype, employing monoclonal antibodies labeled with phycoerythrin (PE), fluorescein isothiocyanate (FITC), or allophycocyanin (APC) and directed against: CD10 (BD Biosciences, San Jose, CA, USA), CD29 (clone TS2/16; eBioscience, San Diego, CA, USA), CD44 (clone IM7; Biolegend, San Diego, CA, USA), CD45 (BD Pharmingen, BD Bioscience, San Jose, CA, USA / Milenyi Biotec, Calderara di Reno, BO, Italy), CD49a (clone TS2/7; Biolegend), CD49b (BD Pharmingen), CD49d (clone 9F10; Biolegend, San Diego, CA, USA), CD49f (clone GOH3; Biolegend, San Diego, CA, USA), CD66e (Serotec, Oxford, UK), CD90 (clone SE10; eBioescience), CD133 (Miltenyi Biotec), CD146 (BD Pharmingen), E-Cadherin (clone 67A4; Biolegend), EGFR (clone AY13; Biolegend), EpCAM (clone 9C4; Biolegend), human epidermal growth factor receptor 2/ HER2 (clone 24D2; Biolegend), and $\mathrm{N}$-Cadherin (clone 8C11; BD Biosciences). Isotype matched antibodies were used as controls. Cells were analyzed employing a FACSCantoII (BD Biosciences) flow cytometer. Differentially expressed antigens were subsequently tested in the $\mathrm{CD} 45^{\text {neg }}$ fraction (CTC enrichment; see later) of healthy donors to exclude those able to bind circulating cells in controls.

\section{Immunomagnetic enrichment and sample staining procedures}

Blood samples $(7.5 \mathrm{ml})$ were first subjected to red blood cell lysis, employing isotonic ammonium-chloride buffer, and then incubated for 20 minutes with CD45 MicroBeads (Miltenyi Biotec). Subsequently, samples were immunomagnetically depleted of the CD $45^{\text {pos }}$ leukocyte fraction using LD separation columns in a MACS MIDI separator (Miltenyi Biotec), according to the manufacturer's instructions. To evaluate the efficiency and the yield of the depletion strategy, both $\mathrm{CD} 45^{\text {neg }}$ and $\mathrm{CD} 45^{\text {pos }}$ fractions were labeled with CD45 and analyzed by fluorescence-activated cell sorting (FACS). CD45 depleted samples were subsequently stained with a cocktail of antibodies recognizing: epithelial markers (i.e., EpCAM, E-Cad) labeled by FITC, mesenchymal markers (i.e., CD44, CD146, and N-Cadherin) labeled by $\mathrm{PE}$, and the pan-leukocyte CD45 marker labeled by APC.

\section{Spiked samples}

For mimicking the in vivo presence of different subsets of CTC in the peripheral blood of MBC patients, known numbers of MCF-7 and MDA-MB231 cells, prelabeled with Hoechst 33342, were spiked into $7.5 \mathrm{ml}$ of blood samples $(n=27)$ obtained from healthy donors. After erythrocyte lysis and immunomagnetic depletion of CD $45^{\text {pos }}$ cells, the enriched samples were stained and analyzed by flow cytometry, as already described, to compute the yield of recovery, sensitivity, and specificity of the entire procedure.

\section{Immunofluorescence analysis of intracellular markers}

For immunofluorescence analysis of intracellular markers, cells were fixed in $4 \%$ buffered paraformaldehyde, and permeabilized with $0.1 \%$ Triton X-100. Estrogen receptor (ER), vimentin, and cytokeratins were detected using the following monoclonal antibodies: anti-ER $\alpha$ (Clone SP1; ACZON, Monte San Pietro, BO, Italy), anti-vimentin (Clone V9; DAKO, Glostrup, Denmark), and anticytokeratins (8, 18, 19; BIO GENEX, San Ramon, CA, USA). Alexa Fluor 555 conjugated secondary antibodies (Invitrogen, Carlsbad, CA, USA) were used. Cells were analyzed by Leica DMI6000 B (Leica Microsystems, Wetzlar, Germany) utilizing a $40 \times$ oil immersion objective (numerical aperture: 1.25).

\section{CTC detection and sorting by the DEPArray}

Blood samples, depleted of CD $45^{\text {pos }}$ cells, were stained with Hoechst 33342 and the afore-described antibody cocktail. Stained cells were resuspended in $14 \mu \mathrm{l}$ of RPMI1640 supplemented with $10 \%$ fetal bovine serum and $1 \%$ antibiotics (all from Invitrogen).

Cell sorting experiments were performed by DEPArray (Silicon Biosystems) as described in the manufacturer's instructions. Briefly, DEPArray cartridges (A300K) were manually loaded with $14 \mu \mathrm{l}$ of sample and $830 \mu \mathrm{l}$ of culture medium. After loading the cartridge into the DEPArray system, the sample was injected by the system into a microchamber where the cells were exposed to an electric field consisting of 16,000 electrical cages in which individual cells are trapped. Image frames for each of the four fluorescent filters (FITC, PE, APC, and 4',6diamidino-2-phenylindole (DAPI)/Hoechst) and brightfield images were captured. Cell detection was based on a DAPI/Hoechst fluorescence threshold. For each cell, a unique ID was assigned. Captured images were processed and presented by the CellBrowser software that enables selection of cells of interest by the operator. Nucleated cells negative for CD45 were chosen, independently from the expression of epithelial and/or mesenchymal markers, and moved to a parking area in the cartridge. Individual cells were then subsequently moved to a recovery area where a last visual confirmation of cell presence could be performed. Typically, the procedure required about 2.5 hours for first recovery, with imaging of four channels including bright-field, and about $15 \mathrm{mi}-$ nutes for the recovery of each cell. 
After DEPArray analysis, single tumor cells were recovered alive and then subjected to transcriptional analysis of target genes by multiplex reverse transcriptase PCR (RT-PCR).

\section{mRNA isolation and quantitative PCR analysis from single cells}

After a single cell lysis step, performed according to the Ampli1 Whole Genome Amplification Kit (Silicon Biosystems) manufacturer's instructions, the mRNA was isolated from the sample and reverse-transcribed into cDNA (Superscript; Invitrogen). All of the reagents required for quantitative RT-PCR were combined in a master mix (Universal SYBR Green Master Mix; Roche, Basel, Switzerland), including primers (Additional file 1: Table S1), probes, and enzyme (according to the manufacturer's instructions). Then $14 \mu \mathrm{l}$ aliquots of this solution and 1 $\mu \mathrm{l}$ of single-cell cDNA were dispensed in a 96-well plate. Real-time PCR and analyses were performed, employing a LightCycler 480 (Roche) instrument. For each amplification reaction, the melting temperature $(\mathrm{Tm})$ and $\mathrm{Cp}$ values were computed.

\section{Statistical analysis}

Patients' characteristics have been summarized by means of descriptive analysis. Categorical variables were described by frequency distribution, whereas continuous variables were reported as median and interquartile range. Age, performance status, and number of lines were dichotomized, according to clinical interest, using respectively 70 years, ECOG PS 1 , and the median of lines received $(n=2)$ as the threshold.

CTC subpopulation distributions were tested for normality by Shapiro-Wilk test and their association with clinicopathological features was explored by Wilcoxon rank-sum test or Kruskal-Wallis test, as appropriate. Owing to the exploratory purpose of the study, no corrections for multiple comparisons were applied. The prognostic impact of CTC count was investigated by univariate Cox regression models with $95 \%$ confidence interval both in terms of overall survival (OS; calculated from both the stage IV diagnosis and the initial CTC assessment) and progression-free survival (PFS; calculated from CTC assessment to the first evidence of disease progression or death). The proportional hazard assumption was tested through the Schoenfeld residuals test.

Differences between CTC distribution quartiles were described by Kaplan-Meier estimator plot and tested by the log-rank test. $p<0.05$ was considered significant. Statistical analysis was conducted using StataCorp 2013 Stata Statistical Software: Release 13 (College Station, Texas, USA).

\section{Results}

Patient recruitment and baseline characteristics

This study enrolled $56 \mathrm{MBC}$ patients treated at the University Hospital of Udine between March 2013 and May 2015, regardless of the line of treatment. CTC assessment was performed before the beginning of a new therapeutic line. Among them, 47 patients were eligible for CTC analysis. Table 1 summarizes patients' clinicopathological characteristics and treatment history. Median age was 62 years (range 36-82). The most

Table 1 Demographic and clinicopathological features of the 47 analyzed metastatic breast cancer patients

Age

Median (range)

$62(36-82)$

$<70$ years

$36(77 \%)$

$\geq 70$ years

$11(23 \%)$

ECOG PS, $n(\%)$

0

$29(62 \%)$

1

$14(29 \%)$

2

$4(9 \%)$

Immunophenotype, $n$ (\%)

Luminal-like

$25(54 \%)$

HER2-positive

$11(23 \%)$

Triple negative

$11(23 \%)$

Histotype, $n$ (\%)

Ductal

$38(84 \%)$

Lobular

$7(16 \%)$

Metastatic sites, ${ }^{a} n(\%)$

Bone

$29(62 \%)$

Liver

$22(47 \%)$

Lung

$16(34 \%)$

CNS

$3(6 \%)$

Number of metastatic sites, $n(\%)$

1

$16(34 \%)$

$\geq 2$

$31(66 \%)$

Number of previous lines, $n$ (\%)

0

$15(32 \%)$

1

$9(19 \%)$

$\geq 2$

$23(49 \%)$

Type of treatments received, $n(\%)$

$\begin{array}{ll}\text { Chemotherapy } & 33(70 \%) \\ \text { Endocrine therapy } & 6(13 \%) \\ \text { Chemotherapy and endocrine therapy } & 5(11 \%) \\ \text { Palliative care } & 2(6 \%) \\ \text { Anti-HER2 targeted therapy } & 12(26 \%)\end{array}$

${ }^{\mathrm{a}}$ Patients may have more than one site involved ECOG PS Eastern Cooperative Oncology Group Performance Status, CNS central nervous system, HER2 human epidermal growth factor receptor 2 
common histotype was ductal (84\%), while the most frequent subtype was luminal-like (54\%). MBC was diagnosed as de novo disease in 15 patients. The median number of previous therapeutic lines was 1 (range $0-10$ ). At the time of recruitment, $66 \%$ of patients presented at least one visceral site of metastatic spread, while $10 \%$ of patients were affected by bone-only disease. At the database lock, disease progression was observed in 29 cases and death in 18 cases. Median follow-up was 33 months, median OS from CTC assessment was 21.7 months, while median PFS was 8.12 months. The estimated 1year and 2-year OS rates were $70 \%$ and $46 \%$, respectively, while the estimated 1-year and 2-year PFS rates were $37 \%$ and $19 \%$ respectively.

\section{Identification of epithelial and mesenchymal CTC by means of surface antigen expression}

A possible limitation of the strategies currently used for the enumeration of CTC from blood samples is the heterogeneous expression of EpCAM on tumor cells, especially on those undergoing EMT [20]. In order to circumvent this issue, we decided to adopt a CTC enrichment strategy based on red blood cell lysis followed by the immunomagnetic depletion of leukocytes from blood samples (i.e., a negative selection; Fig. 1a). The efficiency of this procedure was $99.98 \pm 0.012 \%(n=8$; Fig. 1b) and an average of $2656 \pm 2531$ cells $/ 7.5 \mathrm{ml}$ of processed blood $(n=6)$ could be recovered.

In order to prospectively identify both epithelial-like and mesenchymal-like CTC, we evaluated the surface antigen immunophenotype of two breast cancer lines considered to be prototypical epithelial-like (MCF7) and mesenchymal-like (MDA-MB231) tumor cells [21, 22]. MCF-7 and MDA-MB231 cells differed in the expression of EpCAM, E-Cadherin, CD44, CD49f, and CD146, the first two being upregulated in epithelial-like cells but the latter three in mesenchymal-like cells (Fig. 1c, d). Since our objective was to identify an antibody cocktail able to recognize tumor cells with high specificity, we employed flow cytometry to evaluate the expression of the candidate markers in the CD45-negative fraction of 10 healthy donors. In this way we could exclude those antigens that were commonly present in circulating cells. In fact, while CD49f was frequently observed in donor blood cells, E-Cadherin, EpCAM, CD146, and CD44 were almost undetectable in the CD45-negative fraction of female donors (Fig. 1e). Therefore, we tested, in spiked donor samples subjected to negative selection, an antibody cocktail recognizing epithelial (i.e., FITC-labeled anti-EpCAM, and anti-E-Cad), mesenchymal (i.e. PElabeled anti-CD44, and anti-CD146), and leukocyte (i.e., APC-labeled anti-CD45) markers. This strategy was able to identify, in spiked samples $(n=27)$, Hoechst-labeled breast cancer cells with a sensitivity of $99 \pm 1.4 \%$, a specificity of $99 \pm 0.7 \%$, and a positive predictive value of $95 \pm 5.7 \%$. Values were similar for epithelial-like breast tumor cells $(99.7 \pm 0.4 \%, 98.4 \pm 2 \%, 90 \pm 2.6 \%)$ and mesenchymal-like breast tumor cells $(98 \pm 1.7 \%$, $99.9 \pm 0.1 \%, 99.9 \pm 0.1 \%$ ) (Fig. 1f). To further confirm the specificity of the cocktail, this latter was tested in the CD45-negative fraction of 18 female donors; no epithelial cells were detected, while cells expressing only mesenchymal markers were documented, at low number, in three samples (Additional file 2: Table S2A).

\section{Enumeration and sorting of viable single CTC by DEPArray technology}

We took advantage of the DEPArray system to identify and sort single, viable CTC, based on a multiparametric fluorescence analysis. Specifically, spiked samples containing epithelial-like and/or mesenchymal-like breast tumor cells were stained with Hoechst 33342 and the afore-described antibody cocktail. This approach allowed us to identify and sort single breast tumor cells as viable nucleated cells, negative for CD45 and expressing epithelial and/or mesenchymal markers (Fig. 2a). Sorted cells were suitable for RNA isolation followed by real-time PCR analysis of transcripts typical of epithelial and EMT cells (Fig. 2b).

Once the isolation protocol was optimized, we analyzed blood samples obtained from enrolled patients. In this case, to the afore-described antibody cocktail we added N-Cadherin, a marker of EMT [23] not expressed on human $C D 45^{\text {neg }}$ cells (Fig. 1e). On the basis of the DEPArray analysis, CD45-negative cells were classified as epithelial CTC (expressing only epithelial markers (E CTC)), CTC in EMT (coexpressing epithelial and mesenchymal markers (EM CTC)), putative mesenchymal cells (expressing only mesenchymal markers (MES)), and negative cells (not expressing the tested markers (NEG)). Raw data are presented in Additional file 3: Table S3. All of these cell types could be detected and sorted (Fig. 2c and Table 2). Specifically, the median number of $96 \mathrm{CD} 45^{\text {neg }}$ cells per $7.5 \mathrm{ml}$ of peripheral blood was counted. Of these, more than $2 / 3$ were expressing only mesenchymal markers, $4 \%$ were negative for all of the tested antibodies, and a median fraction of $16 \%$ was positive for the analyzed epithelial markers. Of these latter cells, positive to the epithelial cocktail and therefore considered as bona fide CTC, $22 \%$ coexpressed mesenchymal markers. When the same protocol was applied to blood samples obtained from three female donors, no E CTC, EM CTC, and MES cells were detected (Additional file 2: Table S2B), confirming the absence of bona fide CTC in healthy donors. Conversely, NEG cells were documented in all samples, although at a low number (Additional file 2: Table S2B). 

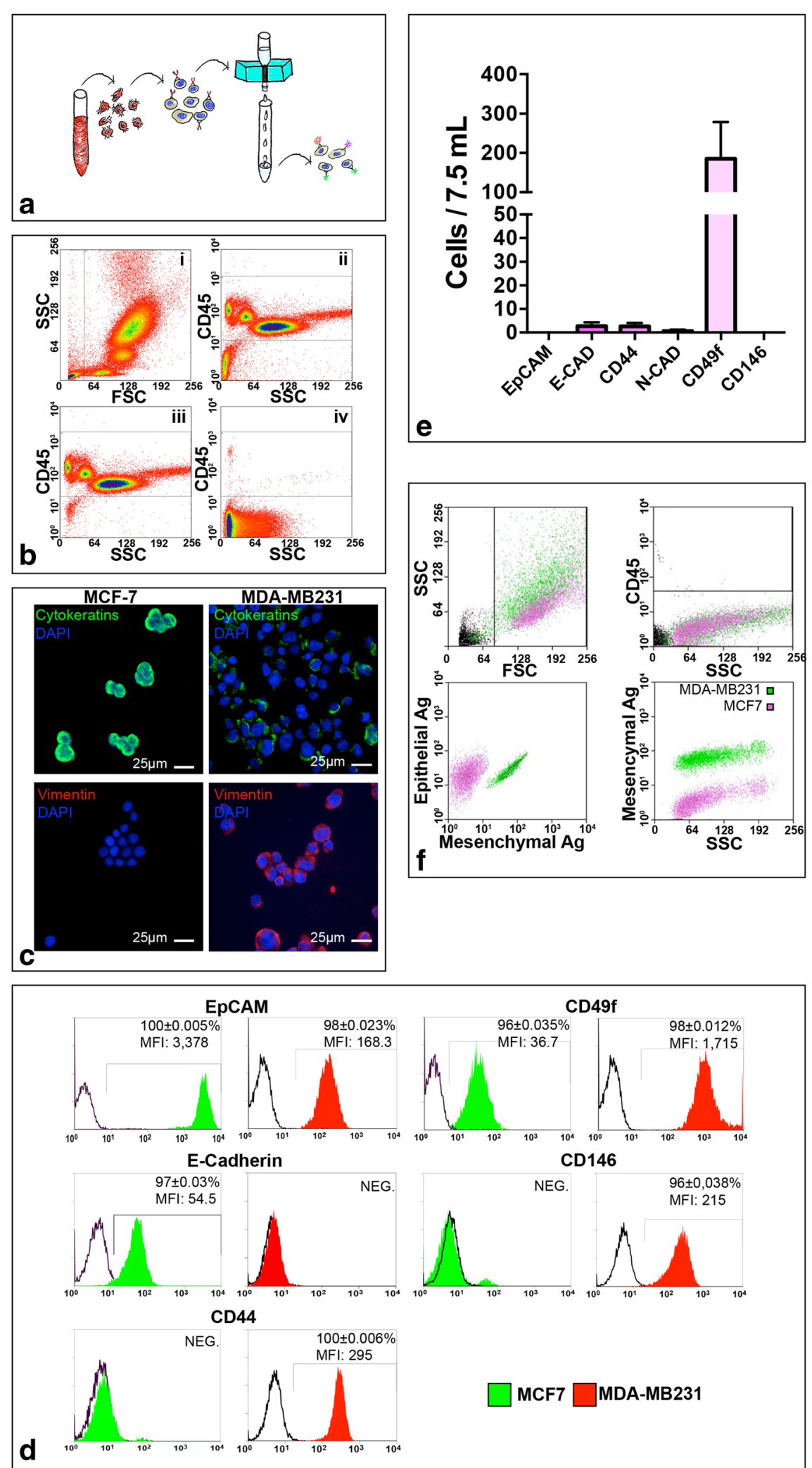

Fig. 1 (See legend on next page.) 
(See figure on previous page.)

Fig. 1 CTC enrichment strategy. a Scheme showing the enrichment strategy. b Dot plots showing physical parameters (i) and CD45 expression of cells before (ii) and after depletion (iii), (iv). CD45-positive (iii) and CD45-negative (iv) fractions are shown. c Immunofluorescence analysis of the epithelial-like (MCF7, left panels) and mesenchymal-like (MDA-MB231, right panels) breast cancer cell lines showing cytokeratin (green fluorescence) and vimentin (red fluorescence) expression. Nuclei are labeled by DAPI (blue fluorescence). d FACS analysis showing, in histograms, the differential expression of epithelial and mesenchymal surface antigens by MCF-7 (green histograms) and MDA-MB231 (red histograms) cell lines. White histograms show negative controls. e Histograms showing the estimated number of CD45-negative peripheral blood mononuclear cells, isolated from $7.5 \mathrm{ml}$ of healthy blood female donors $(n=10)$, expressing either epithelial or mesenchymal antigens. f FACS plot showing the ability of the enrichment strategy to identify MCF7 and MDA-MB231 cells spiked into healthy donor peripheral blood samples. DAPI 4',6-diamidino-2-phenylindole, EpCAM epithelial cell adhesion molecule. (Color figure online), FSC forward scatter, SSC side scatter, MFI mean fluorescence intensity

Downstream mRNA analysis supported the classification based on the surface immmunophenotype. Specifically, single sorted epithelial cells did not express vimentin, while cells in EMT expressed both vimentin and cytokeratin 19, and mesenchymal cells expressed vimentin only. Additionally, in patient-derived E CTC, the expression of HER2 and ER transcripts could also be demonstrated (Fig. 2d).

Altogether these findings indicate that the DEPArraybased CTC detection protocol we optimized could identify CTC subsets in clinical samples obtained from MBC patients. Importantly, the collection of viable, single CTC followed by gene expression analysis corroborates our classification based on cell surface immunophenotype.

\section{Association between CTC subpopulations and clinicopathological features}

Next, we assessed the association between the clinicopathological characteristics of MBC patients and the different subtypes of circulating cells that were identified, as already described, by DEPArray (Table 3, Fig. 3).

Patients affected by HER2-positive disease, with respect to those affected by HER2-negative disease, had a significantly lower number of MES cells $(p=0.022)$, EM CTC (both in absolute number and in percentage, $p=0.029$ and $p=0.035$, respectively), ETOT CTC ( $p=$ $0.037)$, and $C D 45^{\text {neg }}(p=0.046)$. Intriguingly, patients affected by triple-negative disease showed, with respect to those affected by other profiles, a significantly higher proportion and absolute number of NEG cells ( $p=$ 0.024 and $p=0.033$, respectively). The presence of bone metastases was significantly associated with an increase in the absolute number of E CTC $(p=0.0074)$ and EM CTC $(p=0.024)$ and with both the absolute number $(p=0.0058)$ and the percentage $(p=0.00060)$ of CTC expressing epithelial markers (irrespective of the expression of mesenchymal markers (ETOT CTC)). Additionally, bone metastases were associated with a lower percentage of MES cells $(p=0.012)$, while patients with liver localizations were characterized by a higher proportion of E CTC ( $p=0.011)$. Of note, patients with central nervous system involvement were characterized by a higher number of circulating NEG cells, both when considered as absolute numbers $(p=0.016)$ and as a percentage $(p=0.020)$. A higher absolute number of negative cells was also associated with highly proliferating primary breast tumors (Ki67 $\geq 14 \%$; $p=0.039$ ).

Interestingly, even if marginally significant, a lower percentage of MES cells was observed among patients with liver localizations and CNS involvement.

The number of metastatic sites did not seem to influence the number or proportion of different CTC populations.

Altogether these results indicate that important clinical features of MBC patients are associated with distinct subpopulations of circulating cells. Therefore, the indepth characterization of CD $45^{\text {neg }}$ cells may be endowed with a prognostic or predictive significance.

\section{Exploration of the prognostic role of CTC}

Lastly, we evaluated whether the enumeration of CD45 $5^{\text {neg }}$ subpopulations by the DEPArray system could predict outcome.

Univariate Cox regression analysis showed a prognostic role of both ETOT CTC and EM CTC, as a continuous percentage variable, in terms of both OS from stage IV diagnosis ( $p=0.015$ and $p=0.022$, respectively) and from CTC assessment ( $p=0.013$ and $p=0.0016$, respectively). However, the proportion of EM CTC was the only parameter that resulted to be significantly associated with PFS $(p=0.016)$. Intriguingly, the proportion of MES/CD $45^{\text {neg }}$ had a favorable impact in terms of OS (from stage IV diagnosis $p=0.037$ ). The full set of variables investigated in the univariate analysis is reported in Table 4.

Furthermore, we assessed that stratifying the $\mathrm{MBC}$ population according to the percentage of EM CTC and MES could help in describing prognosis. The resulting Kaplan-Meier estimator plots are shown in Fig. 4.

Altogether these results indicate that the identification of the subpopulation of E CTC coexpressing mesenchymal markers may help in discriminating the subset of patients that are at high risk for disease progression.

\section{Discussion}

Over the last 10 years, a general consensus has been reached on the role exerted by the quantitative analysis 

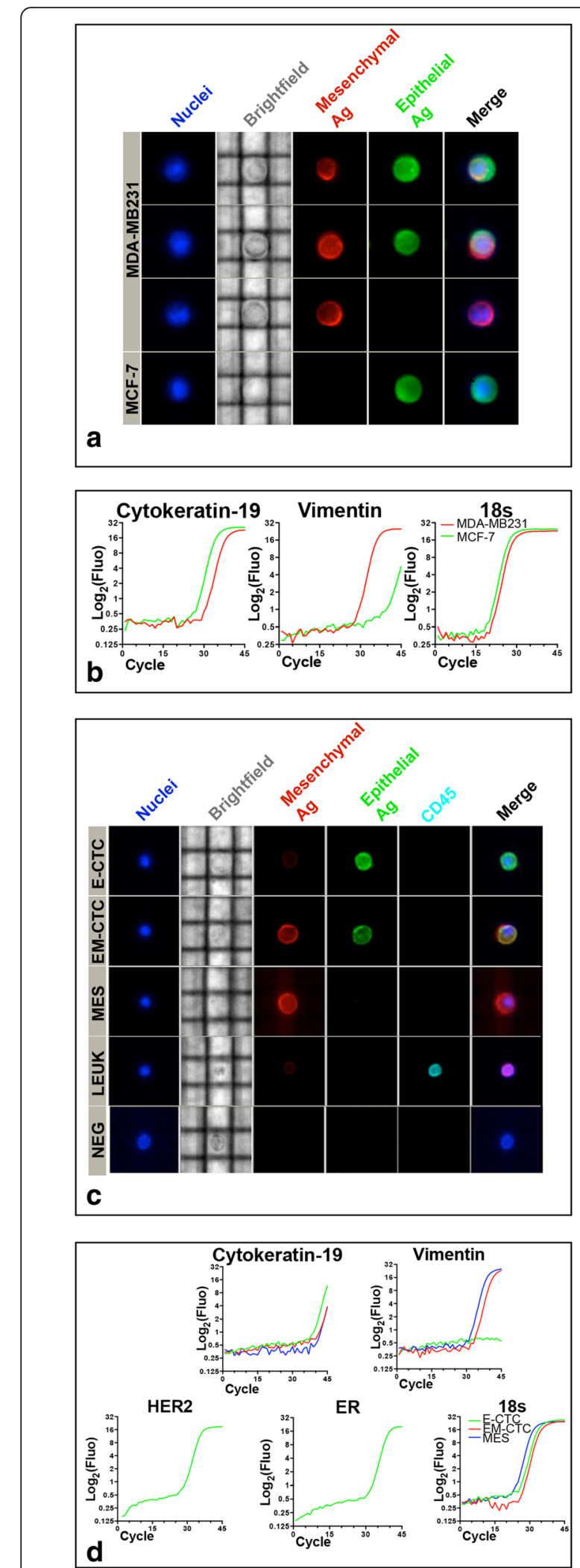

Fig. 2 Enumeration and sorting of viable single CTC by DEPArray technology. a Image gallery of MDA-MB231 and MCF-7 cells, analyzed from a spiked sample, showing, from top to bottom, two cells coexpressing mesenchymal (red fluorescence) and epithelial (green fluorescence) markers, one cell expressing only mesenchymal markers, and one cell expressing only epithelial markers, respectively. Images of tumor cell morphology are shown in the bright-field channel (grey), while cell nuclei are stained by Hoechst (blue). b Real-time PCR showing cytokeratin-19 and vimentin expression in sorted, single MCF-7 (green line) and MDA-MB231 (red line) cells. c Representative images of the analysis of a blood sample from a MBC patient enriched employing the strategy described in Fig. 1a. The CD45-depleted fraction was labeled with antibodies recognizing epithelial (green fluorescence) and mesenchymal (red fluorescence) markers, as well as the common leukocyte antigen CD45 (cyan fluorescence). The image bar displays, from top to bottom, one cell expressing only epithelial markers (E CTC), one cell coexpressing mesenchymal and epithelial markers (EM CTC), one cell expressing only mesenchymal markers (MES), one CD45-positive leukocyte (LEUK), and one cell negative for all of the tested markers (NEG), respectively. Images of tumor cell morphology are shown in the bright-field channel (grey), while cell nuclei are stained by Hoechst (blue). d Real-time PCR showing cytokeratin-19, vimentin, HER2, and ER expression in sorted, single E-CTC (green line), EM-CTC (red line), and MES (blue line) cells (Color figure online)

of CTC in the prognostic stratification of patients with MBC $[1,5,8]$. As a consequence, it has been suggested to employ CTC as a liquid biopsy to perform real-time monitoring of the metastatic status of a given patient [24], possibly offering an important parameter for precision medicine and personalized treatments [25].

The only FDA-approved instrument for the enumeration of CTC is, thus far, the CellSearch System (Veridex) [26]. Although this latter is robust and its ability to predict patients' prognosis has been validated over the years $[5,8,27]$, several potential limitations have been described. Specifically, the CellSearch System processes fixed blood samples and selects CTC from blood cells relying on the expression of EpCAM on their surface. However, several investigators [28, 29] have raised concerns regarding the homogeneous expression of this antigen on CTC. This issue is especially prominent in cells undergoing EMT, a process that downregulates typical epithelial markers (such as EpCAM, E-Cadherin, and keratins), upregulates mesenchymal markers (e.g. vimentin), and is characterized by reduced adhesive properties and increased invasiveness [20]. To overcome this major intrinsic limitation of the methodology, in recent years several investigators have tried to develop alternative assays that either target antigens alternative to EpCAM (e.g., CD146, CD49f) [29-31] or take advantage of different, antigen-independent, techniques (e.g., PCR-based assays, density gradient centrifugation, cell filtration) [1, 20]. However, the latter techniques, still at an experimental stage, are not approved for diagnostic purposes because they lack a clinical validation [14]. 
Table $2 \mathrm{CD}^{\mathrm{n}} 5^{\text {neg }}$ subpopulations at baseline

\begin{tabular}{|c|c|c|}
\hline Cell class & Immunophenotype & Median $\left(25^{\text {th }} ; 75^{\text {th }}\right)$ \\
\hline$E C T C^{a}$ & $\mathrm{E}^{+} \mathrm{M}^{-}$ & $8(3 ; 24)$ \\
\hline EM CTC ${ }^{a}$ & $E^{+} M^{+}$ & $3(0 ; 9)$ \\
\hline $\mathrm{MES}^{\mathrm{a}}$ & $E^{-} M^{+}$ & $45(15 ; 95)$ \\
\hline $\mathrm{NEG}^{\mathrm{a}}$ & $E^{-} M^{-}$ & $4(0 ; 14)$ \\
\hline Derived parameters & Function & Median $\left(25^{\text {th }} ; 75^{\text {th }}\right)$ \\
\hline $\mathrm{E}^{\mathrm{TOT}} \mathrm{CTC}^{\mathrm{a}}$ & $\mathrm{EM}$ CTC + E CTC & $10(4 ; 33)$ \\
\hline $\operatorname{CD} 45^{\text {nega }}$ & ETOT CTC + MES + NEG & $96(42 ; 145)$ \\
\hline EM CTC fraction over CD45 neg b & $\mathrm{EM}$ CTC/CD45 $5^{\text {neg }} \times 100$ & $2(0 ; 8)$ \\
\hline ETOT CTC fraction over CD45 neg b & ETOT CTC/CD45 $5^{\text {neg }} \times 100$ & $16(5 ; 30)$ \\
\hline MES fraction over CD $45^{\text {neg } b}$ & $\mathrm{MES} / \mathrm{CD} 45^{\mathrm{neg}} \times 100$ & $79(40 ; 88)$ \\
\hline NEG fraction over CD45 $5^{\text {neg } b}$ & $\mathrm{NEG} / \mathrm{CD} 45^{\text {neg }} \times 100$ & $4(0 ; 15)$ \\
\hline
\end{tabular}

Summary of the definition of the cell subpopulations counted by DEPArray, the parameters computed from these absolute cell numbers, and the descriptive statistics, expressed as median and interquartile range, of cell classes and derived parameters $(n=47)$

${ }^{\text {a}}$ Results expressed as absolute number of cells for $7.5 \mathrm{ml}$ of peripheral blood

${ }^{\mathrm{b}}$ Results expressed as percentage

$E$ reactivity to the epithelial antibody cocktail, ECTC subset of CD45 ${ }^{\text {neg }}$ expressing only epithelial markers, EM CTC subset of CD45 ${ }^{\text {neg }}$ coexpressing mesenchymal and epithelial markers, ETOT CTC CD $45^{\text {neg }}$ cells expressing epithelial markers independently from the expression of mesenchymal markers, $M$ reactivity to the mesenchymal antibody cocktail, MES subset of $C D 45^{\text {neg }}$ expressing mesenchymal markers only, NEG subset of CD45 ${ }^{\text {neg }}$ negative to the antibody cocktail

In this work, we have optimized an alternative enrichment strategy, taking into account that, as previously stated, the "perfect" CTC marker should be expressed on all CTC, but not on autochthonous blood cells [1]. Therefore, we first depleted leukocytes employing anti-CD45 immunomagnetic beads. Subsequently, we selected the antibody cocktail that showed the best performance in discriminating epithelial and mesenchymal surface antigens on CTC. For this purpose we screened 17 antigens to identify those that were differentially expressed by a luminal-like cell line (i.e., MCF7) and a triple negative-like cell line (i.e., MDA-MB231). Candidate antigens were further refined to exclude those that were expressed on circulating, CD $45^{\text {neg }}$ cells, in female blood donors. This way we decided that, as opposed to a previous report [31], CD49f was not a useful marker in our hands. Employing this strategy, we were able to sort with high reproducibility breast cancer cells from spiked samples.

Another possible limitation of the CellSearch System is its inability to sort single, viable, and unfixed CTC to perform further downstream analyses. To circumvent this problem, we employed a dielectrophoresis-based system [32] (i.e., the DEPArray system) that has the ability to perform fluorescence microscopy analysis, mobilization, and collection of single viable cells from a pool of up $\approx 100,000$ cells. In our hands, the labeling strategy that we optimized was a perfect match for the DEPArray system. In fact, the immunodepletion approach resulted in a number of recovered cells that was compatible with the loading capacity of the DEPArray chip (but avoids the loss of cells that do not express EpCAM), and the multiparametric analysis, based on an antibody cocktail recognizing both epithelial and mesenchymal antigens, allowed the identification of different cell subtypes. Last, our approach permitted us to isolate single, viable CTC [33] that could be further analyzed by quantitative RT-PCR. As a result, however, our method of analysis is slower than the method employed by the CellSearch System. In fact, with the workflow for sample preparation and analysis suggested in the manuscript, two patients per day can be processed. However, we can obtain, for each analyzed patient, an improved evaluation of the CTC heterogeneity, and molecular studies of single sorted CTC might add valuable prognostic and predictive information. By systematically applying our analysis to 47 blood samples obtained from MBC patients, we observed four different cell subpopulations: CTC expressing only epithelial antigens, CTC coexpressing epithelial and mesenchymal antigens, cells expressing only mesenchymal antigens, and cells that do not express either epithelial or mesenchymal antigens. While the first two cell types are obviously CTC, less clear is the neoplastic nature of the latter two cell types. Ongoing single-cell genomic analysis experiments will clarify this issue (data not shown).

However, the more in-depth analysis of the CD $45^{\text {neg }}$ fraction has allowed us to identify specific circulating cell subsets that are significantly associated with relevant clinical parameters. In fact, we showed that different breast tumor subtypes are associated with a distinct pattern of circulating $\mathrm{CD} 45^{\text {neg }}$ cell subpopulations. Additionally, we observed that HER2-positive tumors were characterized by a trend to a reduced number of circulating MES and ETOT CTC. This finding is in line with data obtained by Cristofanilli's group [34] and could 
Table 3 Association between circulating CD45 ${ }^{\text {neg }}$ subpopulations and clinicopathological features of MBC patients

\begin{tabular}{|c|c|c|c|c|c|c|c|c|c|c|c|}
\hline & & \multicolumn{6}{|c|}{ Absolute number } & \multicolumn{4}{|c|}{ Fraction over CD45 ${ }^{\text {neg }}(\%)$} \\
\hline & & E CTC & EM CTC & ETOT CTC & MES & NEG & CD45 $5^{\text {neg }}$ & EM CTC & ETOT CTC & MES & NEG \\
\hline \multicolumn{12}{|l|}{ Tumor characteristics } \\
\hline \multirow[t]{3}{*}{ HER2 } & Positive $(N=11)$ & $3(0 ; 9)$ & $0(0 ; 3)$ & $3(1 ; 12)$ & $22(6 ; 42)$ & $0(0 ; 14)$ & $42(19 ; 118)$ & $0(0 ; 3)$ & $16(4 ; 25)$ & $81(33 ; 97)$ & $0(0 ; 38)$ \\
\hline & Negative $(N=36)$ & $8(4 ; 27)$ & $4(0 ; 13)$ & $15(5 ; 35)$ & $80(15 ; 124)$ & $4(0 ; 13)$ & $102(60 ; 153)$ & $4(0 ; 14)$ & $17(6 ; 39)$ & $74(40 ; 88)$ & $4(0 ; 15)$ \\
\hline & $p^{*}$ & 0.12 & 0.029 & 0.037 & 0.022 & 0.84 & 0.046 & 0.035 & 0.37 & 0.70 & 0.94 \\
\hline \multirow[t]{4}{*}{ Profile } & Luminal $(N=25)$ & $8(4 ; 32)$ & $4(1 ; 15)$ & $16(6 ; 53)$ & $72(14 ; 103)$ & $0(0 ; 9)$ & $97(59 ; 197)$ & $4(0 ; 14)$ & $19(6 ; 55)$ & $70(39 ; 90)$ & $0(0 ; 6)$ \\
\hline & HER2-positive $(N=11)$ & $3(0 ; 9)$ & $0(0 ; 3)$ & $3(1 ; 12)$ & $22(6 ; 42)$ & $0(0 ; 14)$ & $42(19 ; 118)$ & $0(0 ; 3)$ & $16(4 ; 25)$ & $81(33 ; 96)$ & $0(0 ; 38)$ \\
\hline & TNBC $(N=11)$ & $9(2 ; 24)$ & $3(0 ; 9)$ & $15(2 ; 30)$ & $84(29 ; 125)$ & $9(5 ; 18)$ & $124(60 ; 150)$ & $2(0 ; 8)$ & $16(3 ; 21)$ & $79(60 ; 86)$ & $15(4 ; 16)$ \\
\hline & $p^{* *}$ & 0.30 & 0.044 & 0.087 & 0.052 & 0.092 & 0.12 & 0.064 & 0.35 & 0.93 & 0.056 \\
\hline \multirow[t]{3}{*}{$\mathrm{Ki}-67$} & $<14 \%(N=10)$ & $7(4 ; 35)$ & $7(0 ; 18)$ & $18(6 ; 53)$ & $42(6 ; 103)$ & $0(0 ; 4)$ & $76(41 ; 197)$ & $4(0 ; 31)$ & $19(8 ; 63)$ & $63(37 ; 90)$ & $0(0 ; 5)$ \\
\hline & $\geq 14 \%(N=34)$ & $8(2 ; 16)$ & $2(0 ; 6)$ & $10(3 ; 30)$ & $55(20 ; 92)$ & $8(0 ; 18)$ & $99(42 ; 138)$ & $2(0 ; 7)$ & $16(5 ; 25)$ & $77(41 ; 86)$ & $8(0 ; 16)$ \\
\hline & $p^{*}$ & 0.45 & 0.15 & 0.37 & 0.64 & 0.039 & 0.74 & 0.33 & 0.39 & 0.90 & 0.087 \\
\hline \multicolumn{12}{|l|}{ Metastatic site } \\
\hline \multirow[t]{3}{*}{ Bone } & Yes $(N=29)$ & $10(4 ; 32)$ & $4(0 ; 15)$ & $20(7 ; 52)$ & $45(14 ; 84)$ & $2(0 ; 12)$ & $95(48 ; 126)$ & $5(0 ; 14)$ & $25(14 ; 55)$ & $60(33 ; 81)$ & $2(0 ; 14)$ \\
\hline & No $(N=29)$ & $4(1 ; 6)$ & $0(0 ; 4)$ & $5(1 ; 12)$ & $55(20 ; 129)$ & $6(0 ; 18)$ & $89(23 ; 150)$ & $0(0 ; 2)$ & $7(3 ; 16$ & $85(75 ; 90)$ & $6(0 ; 16)$ \\
\hline & $p^{*}$ & 0.0072 & 0.024 & 0.0058 & 0.34 & 0.43 & 0.97 & 0.0096 & 0.0006 & 0.012 & 0.48 \\
\hline \multirow[t]{3}{*}{ Liver } & Yes $(N=22)$ & $9(4 ; 27)$ & $3(0 ; 9)$ & $15(4 ; 45)$ & $42(15 ; 75)$ & $6(0 ; 14)$ & $90(41 ; 126)$ & $3(0 ; 14)$ & $25(10 ; 55)$ & $63(33 ; 84)$ & $4(0 ; 15)$ \\
\hline & No $(N=25)$ & $6(1 ; 10)$ & $3(0 ; 9)$ & $10(2 ; 21)$ & $84(20 ; 123)$ & $3(0 ; 12)$ & $103(42 ; 156)$ & $2(0 ; 6)$ & $9(3 ; 19)$ & $81(58 ; 89)$ & $2(0 ; 15)$ \\
\hline & $p^{*}$ & 0.28 & 0.99 & 0.27 & 0.28 & 0.87 & 0.47 & 0.48 & 0.011 & 0.067 & 0.86 \\
\hline \multirow[t]{3}{*}{ Lung } & Yes $(N=16)$ & $9(4 ; 20)$ & $1(0 ; 6)$ & $10(6 ; 30)$ & $57(23 ; 126)$ & $6(0 ; 13)$ & $87(42 ; 153)$ & $3(0 ; 14)$ & $12(4 ; 30)$ & $79(49 ; 87)$ & $5(0 ; 16)$ \\
\hline & No $(N=31)$ & $6(2 ; 27)$ & $3(0 ; 15)$ & $10(3 ; 45)$ & $45(10 ; 92)$ & $3(0 ; 14)$ & $95(23 ; 135)$ & $0(0 ; 6)$ & $17(6 ; 33)$ & $75(33 ; 89)$ & $2(0 ; 15)$ \\
\hline & $p^{*}$ & 0.69 & 0.23 & 0.86 & 0.26 & 0.71 & 0.72 & 0.16 & 0.36 & 0.50 & 0.74 \\
\hline \multirow[t]{3}{*}{ CNS } & Yes $(N=3)$ & $24(8 ; 47)$ & $6(0 ; 21)$ & $45(8 ; 53)$ & $45(30 ; 75)$ & $43(14 ; 90)$ & $126(97 ; 180)$ & $6(0 ; 12)$ & $25(6 ; 55)$ & $31(25 ; 60)$ & $34(14 ; 50)$ \\
\hline & No $(N=44)$ & $6(2 ; 20)$ & $3(0 ; 9)$ & $10(3 ; 32)$ & $53(15 ; 100)$ & $3(0 ; 12)$ & $85(37 ; 143)$ & $2(0 ; 10)$ & $16(5 ; 32)$ & $79(40 ; 89)$ & $2(0 ; 15)$ \\
\hline & $p^{*}$ & 0.16 & 0.56 & 0.26 & 1.0000 & 0.016 & 0.26 & 0.75 & 0.51 & 0.082 & 0.020 \\
\hline \multirow[t]{3}{*}{ Number of sites } & $1(N=16)$ & $5(2 ; 9)$ & $1(0 ; 10)$ & $7(2 ; 17)$ & $55(13 ; 93)$ & $1(0 ; 9)$ & $82(41 ; 122)$ & $2(0 ; 9)$ & $9(3 ; 21)$ & $82(47 ; 91)$ & $1(0 ; 14)$ \\
\hline & $\geq 2(N=31)$ & $9(3 ; 27)$ & $3(0 ; 9)$ & $15(4 ; 36)$ & $45(15 ; 123)$ & $5(0 ; 14)$ & $97(41 ; 156)$ & $2(0 ; 12)$ & $19(6 ; 36)$ & $70(37 ; 86)$ & $5(0 ; 16)$ \\
\hline & $p^{*}$ & 0.22 & 0.76 & 0.19 & 0.84 & 0.40 & 0.67 & 0.78 & 0.16 & 0.28 & 0.61 \\
\hline
\end{tabular}

Results are expressed as median (25th, 75th percentile). Bold cells indicate significant $(p<0.05)$ differences as detected by Wilcoxon rank-sum test $\left.{ }^{*}\right)$ or Kruskal-Wallis test $(* *)$, as appropriate
$E C T C$ subset of $C D 45^{\text {neg }}$ expressing epithelial markers only, EM CTC subset of CD45 ${ }^{\text {neg }}$ coexpressing mesenchymal and epithelial markers, ETOT CTC CD45
neg cells expressing epithelial markers independently from the $E C T C$ subset of $C D 45^{\text {neg }}$ expressing epithelial markers only, EM CTC subset of CD45 $5^{\text {neg }}$ coexpressing mesenchymal and epithelial markers, ETOT CTC CD45neg cells expressing epithelial markers independently from the
expression of mesenchymal markers, MBC metastatic breast cancer, MES subset of CD45 neg expressing mesenchymal markers only, NEG subset of CD45 neg negative to the antibody cocktail, CNS central nervous system, HER2 human epidermal growth factor receptor 2 

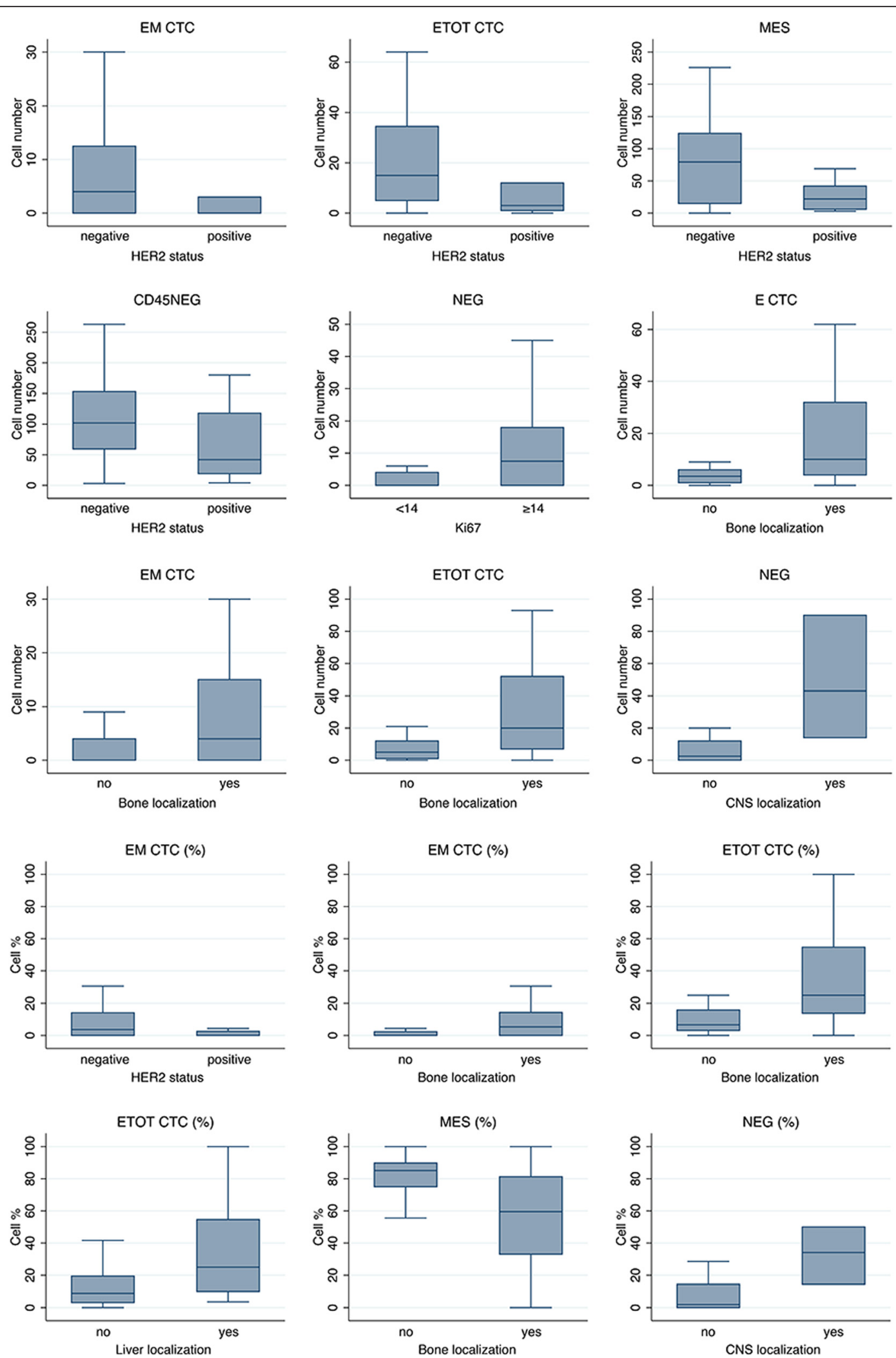

Fig. 3 (See legend on next page.) 
(See figure on previous page.)

Fig. 3 Association between CTC subpopulations and clinicopathological characteristics. Box and whiskers plots showing median, interquartile ranges, 5th and 95th percentile of the absolute number or frequency of different classes of cells (E, E/M, MES, NEG) significantly associated with specific clinicopathological features. E CTC cells expressing only epithelial markers, EM CTC cells coexpressing mesenchymal and epithelial markers, ETOT CTC CD $45^{\text {neg }}$ cells expressing epithelial markers, independently from the expression of mesenchymal markers, MES cells expressing only mesenchymal markers, NEG cells negative for all of the tested markers, CNS central nervous system, HER2 human epidermal growth factor receptor 2

possibly be related to the targeted therapy to which the MBC patient population is exposed. In fact, it has been shown that herceptin can downregulate the expression of chemokine (C-X-C motif) receptor 4 (CXCR4), which is required for HER2-enhanced invasion, migration, and metastasis [35]. Conversely, triple-negative tumors, with respect to the luminal ones, were associated with an increased absolute number and relative number of circulating cells not expressing either mesenchymal or epithelial markers. This evidence suggests that a fraction of CTC not recognized by the antibody cocktail that we employed may be comprised in this cell subset. Importantly, our approach allowed us to sort this cell population, which is now available for downstream molecular analyses. Ongoing DNA sequencing experiments will verify the presence of mutations shared with the tumor of origin, while future gene expression profiling experiments will help in selecting additional surface proteins that could be used to further refine our procedure.
Concerning the association between metastatic sites and the $\mathrm{CD} 45^{\text {neg }}$ cell subsets, an increased number of NEG cells was significantly associated with the presence of brain secondary lesions. This result is in line with literature data showing that CTC with brain metastatic potential are indeed EpCAM-negative [4] and supports the importance of going beyond the pure enumeration of CTC expressing this antigen. Conversely, bone metastases were strongly associated with the absolute and relative abundance of CTC expressing epithelial antigens. This finding is consistent with the observed association of bone metastases with a CTC number $\geq 5$ cells $/ 7.5 \mathrm{ml}$ of peripheral blood, as estimated by CellSearch [36].

Last, we assessed whether the identification of CTC in EMT was endowed with a prognostic significance. Specifically, the fraction of $\mathrm{CD} 45^{\text {neg }}$ cells coexpressing epithelial and mesenchymal markers was associated with both computed patient PFS and OS, the latter both from the diagnosis of stage IV disease and from the CTC

Table 4 Univariate analysis of demographic data, clinicopathological data, and circulating CD45 ${ }^{\text {neg }}$ subpopulations with PFS and OS, computed from both the diagnosis of metastasis and the CTC assessment

\begin{tabular}{|c|c|c|c|c|c|c|c|c|c|c|}
\hline \multicolumn{2}{|l|}{ Variable } & \multicolumn{3}{|c|}{ OS (from stage IV diagnosis) } & \multicolumn{3}{|c|}{ OS (from CTC assessment) } & \multicolumn{3}{|l|}{ PFS } \\
\hline & & $\overline{\mathrm{HR}}$ & $95 \% \mathrm{Cl}$ & $p$ & $\mathrm{HR}$ & $95 \% \mathrm{Cl}$ & $p$ & $\overline{H R}$ & $95 \% \mathrm{Cl}$ & $p$ \\
\hline ECOG PS & $2-3$ vs. $0-1$ & 5.288 & $1.392-20.096$ & 0.015 & 11.294 & $2.467-51.702$ & 0.0018 & 2.558 & $0.730-8.963$ & 0.14 \\
\hline \multirow[t]{2}{*}{ BC profile } & HER2-positive vs. luminal & 0.338 & $0.075-1.525$ & 0.16 & 0.274 & $0.061-1.235$ & 0.092 & 0.616 & $0.237-1.599$ & 0.32 \\
\hline & TNBC vs. luminal & 1.803 & $0.564-5.771$ & 0.32 & 0.972 & $0.312-3.029$ & 0.96 & 1.212 & $0.495-2.963$ & 0.67 \\
\hline Lines received & $>2$ vs. $<2$ & 3.452 & $1.138-10.475$ & 0.029 & 0.622 & $0.245-1.581$ & 0.32 & 0.557 & $0.265-1.172$ & 0.12 \\
\hline Age & $\geq 70$ vs. $<70$ & 0.593 & $0.19-1.809$ & 0.34 & 0.652 & $0.213-1.997$ & 0.45 & 1.031 & $0.459-2.314$ & 0.94 \\
\hline \multicolumn{2}{|l|}{$\mathrm{E} C \mathrm{CT}(n)$} & 1.003 & $0.982-1.024$ & 0.79 & 1.001 & $0.980-1.023$ & 0.92 & 1.004 & $0.989-1.020$ & 0.6 \\
\hline \multicolumn{2}{|l|}{$\mathrm{EM} C \mathrm{CTC}(n)$} & 1.018 & $0.994-1.043$ & 0.13 & 1.023 & 0.998-1.049 & 0.08 & 1.020 & $0.998-1.043$ & 0.081 \\
\hline \multicolumn{2}{|l|}{ ETOT CTC $(n)$} & 1.006 & 0.993-1.019 & 0.35 & 1.006 & 0.993-1.019 & 0.38 & 1.007 & $0.996-1.018$ & 0.24 \\
\hline \multicolumn{2}{|l|}{ MES (n) } & 0.998 & $0.991-1.005$ & 0.56 & 0.998 & $0.991-1.005$ & 0.56 & 0.998 & $0.992-1.003$ & 0.41 \\
\hline \multicolumn{2}{|l|}{$\operatorname{NEG}(n)$} & 0.998 & $0.981-1.015$ & 0.79 & 0.992 & $0.975-1.010$ & 0.41 & 0.997 & $0.986-1.008$ & 0.58 \\
\hline \multicolumn{2}{|l|}{$\mathrm{CD} 45^{\text {neg }}(n)$} & 0.999 & $0.994-1.004$ & 0.79 & 0.999 & $0.993-1.004$ & 0.61 & 0.999 & $0.995-1.003$ & 0.56 \\
\hline \multicolumn{2}{|c|}{ EM CTC/CD45 $5^{\text {neg }}(\%)$} & 1.022 & $1.003-1.042$ & 0.022 & 1.035 & $1.013-1.057$ & 0.0016 & 1.021 & $1.004-1.039$ & 0.016 \\
\hline \multicolumn{2}{|c|}{ E TOT/CD45 neg $(\%)$} & 1.019 & $1.004-1.034$ & 0.015 & 1.019 & $1.004-1.034$ & 0.013 & 1.010 & $0.997-1.023$ & 0.12 \\
\hline \multicolumn{2}{|c|}{ MES/CD45 ${ }^{\text {neg }}(\%)$} & 0.984 & $0.968-0.999$ & 0.037 & 0.988 & $0.973-1.002$ & 0.1 & 0.993 & $0.981-1.005$ & 0.26 \\
\hline \multicolumn{2}{|c|}{ NEG/CD45 ${ }^{\text {neg }}(\%)$} & 0.999 & $0.972-1.027$ & 0.95 & 0.992 & $0.966-1.018$ & 0.54 & 0.998 & $0.980-1.017$ & 0.86 \\
\hline
\end{tabular}

Bold cells indicate significant $(p<0.05)$ association as detected by univariate Cox regression model

Cl confidence interval, CTC circulating tumor cells, ECOG PS Eastern Cooperative Oncology Group Performance Status, E CTC subset of CD45 ${ }^{\text {neg }}$ expressing epithelial markers only, EM CTC subset of CD45 ${ }^{\text {neg }}$ coexpressing mesenchymal and epithelial markers, ETOT CTC CD $45^{\text {neg }}$ cells expressing epithelial markers independently from the expression of mesenchymal markers, $H R$ hazard ratio, MES subset of CD $45^{\text {neg }}$ expressing mesenchymal markers only, NEG subset of $C D 45^{\text {neg }}$ negative to the antibody cocktail, OS overall survival, PFS progression-free survival, BC breast cancer, HER2 human epidermal growth factor receptor 2, TNBC triple negative breast cancer 

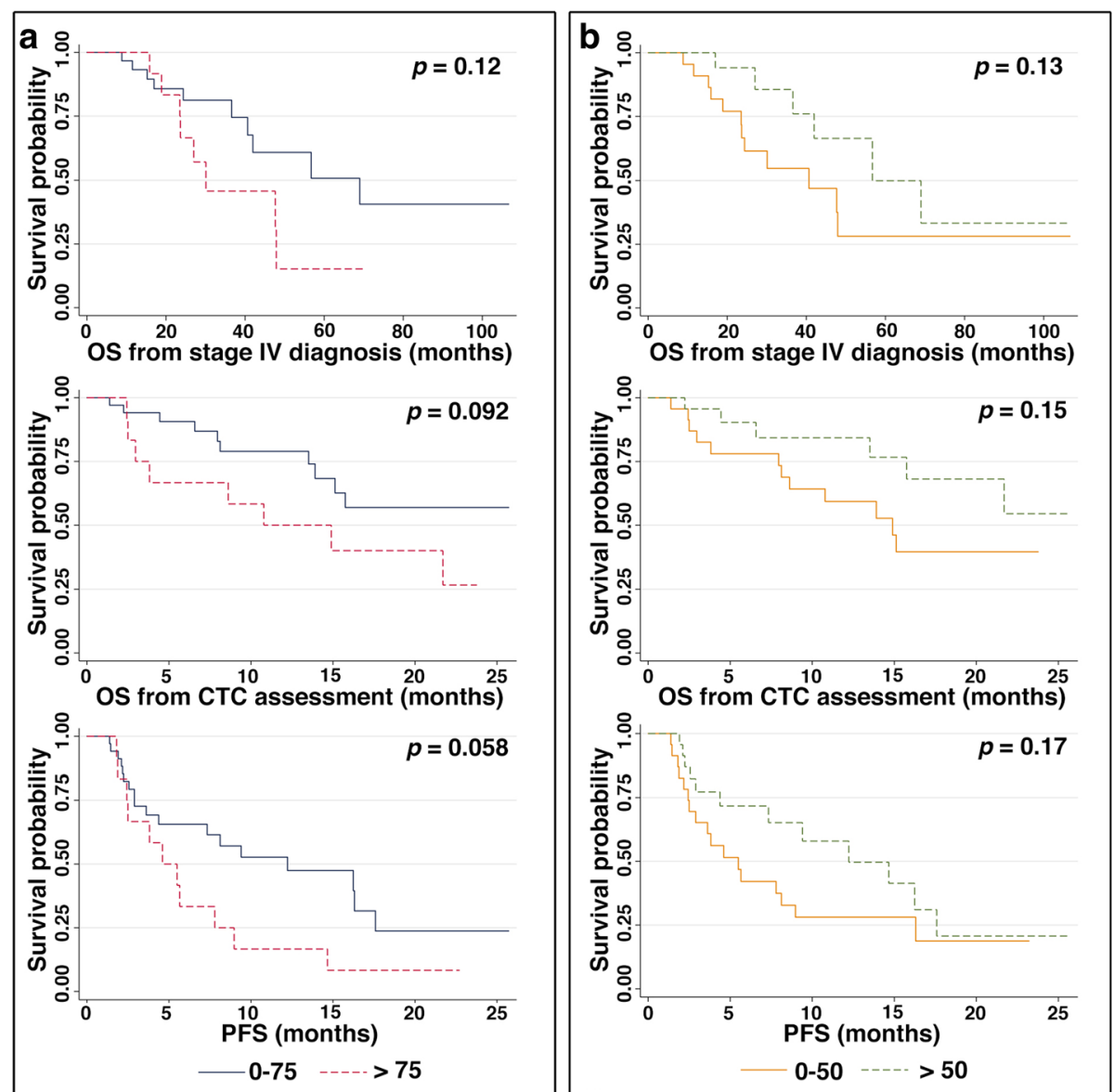

Fig. 4 Survival curves. a Kaplan-Meier estimator plots in terms of OS from stage IV diagnosis (upper panel), OS from CTC assessment (middle panel), and PFS (lower panel) in patients with MBC for those with a fraction of EM CTC above the $75^{\text {th }}$ percentile (dotted red line) and for those with a fraction of EM CTC less than or equal to the $75^{\text {th }}$ percentile (solid black line) before initiation of a new line of therapy. $\mathbf{b}$ Kaplan-Meier estimates in terms of OS from stage IV diagnosis (upper panel), OS from CTC assessment (middle panel), and PFS (lower panel) in patients with MBC for those with a fraction of MES above the 50th percentile (dotted green line) and for those with a fraction of MES less than or equal to the 50th percentile (solid orange line) before initiation of a new line of therapy. CTC circulating tumor cells, OS overall survival, PFS progression-free survival. (Color figure online)

assessment. We decided to evaluate the overall survival from both enrollment into the study and from the diagnosis of metastasis, because, conceptually, these are the expression of two different views. The first considers the cancer as characterized by a site-dependent and timerelated tumor heterogeneity that can be caused by both intrinsic (tumor-related) and extrinsic (therapy-related) factors [37], and CTC analyses have been considered a way to perform real-time monitoring of the metastatic disease by means of a minimally invasive technique (liquid biopsy) [20]. Upon the second view, CTC measurements could predict the intrinsic drug resistance of the metastatic disease. In our prospective observational study, the ability of CTC in EMT to predict the OS from the diagnosis of the metastatic disease suggests the existence of a subset of tumors whose prognosis is not significantly modified by currently available therapies. A similar conclusion has been reached by the clinical study SWOG S0500 [38], where early switching to an alternate cytotoxic therapy in patients with persistently increased CTC after 21 days of first-line chemotherapy was not effective in prolonging OS. Those authors suggested that it would be more profitable for this population of patients to be recruited into prospective trials of novel therapies and to take advantage of molecular analyses of metastasis, CTC, or circulating cell-free DNA to guide therapy [38].

It is important to underline that because of the relatively small sample size, the results from this observational study must be treated cautiously. Accordingly, validation of these findings in a larger independent cohort of patients is needed. 


\section{Conclusions}

In this work we optimized a novel strategy to enrich blood samples in CTC, independent from the expression of epithelial markers. Moreover, taking advantage of the DEPArray system, we have identified and sorted, based on multiparametric fluorescence analysis, single, viable epithelial-like CTC as well as CTC in EMT. Intriguingly, our CTC enrichment strategy allowed us to observe two additional circulating subsets in the $C D 45^{\text {neg }}$ fraction: one expressing only mesenchymal surface proteins, and the other negative for all of the tested markers. The neoplastic nature of these cell populations is still an open issue. Nonetheless, each of these CD $45^{\text {neg }}$ cell subsets was associated, in a prospective study including 47 patients affected by $\mathrm{MBC}$, with clinically relevant parameters, such as tumor subtype, Ki67 expressions and the site of metastasis. Most importantly, the fraction of CTC expressing EMT markers was an independent predictor of poor outcome.

Altogether these results indicate that the protocol that we optimized for the analysis and sorting of circulating $\mathrm{CD} 45^{\text {neg }}$ cells in $\mathrm{MBC}$ patients could be of high clinical relevance and deserves validation in a larger independent cohort of patients.

\section{Additional files}

Additional file 1: Table S1. Presenting primers pairs for real-time PCR. (DOCX $14 \mathrm{~kb}$ )

Additional file 2: Table S2A. Presenting enumeration of different CD45 ${ }^{\text {neg }}$ subgroups, as assessed by FACS, in blood samples obtained from healthy female donors, and Table S2B. presenting enumeration of different CD45 ${ }^{\text {neg }}$ subgroups, as assessed by DEPArray, in blood samples obtained from healthy female donors. (DOCX $90 \mathrm{~kb}$ )

Additional file 3: Table S3. Presenting the CTC count at baseline in each analyzed patient. (DOCX 18 kb)

\section{Abbreviations}

APC: Allophycocyanin; CD45 ${ }^{\text {neg }}$ : circulating cells not expressing CD45; CTC: Circulating tumor cells; CXCR4: Chemokine (C-X-C motif) receptor 4; DAPI: 4',6-Diamidino-2-phenylindole; E CTC: Subset of CD45 ${ }^{\text {neg }}$ expressing epithelial markers only; ECOG PS: Eastern Cooperative Oncology Group Performance Status; EMT: Epithelial-to-mesenchymal transition; EpCAM: Epithelial cell adhesion molecule; ER: Estrogen receptor; ETOT CTC: CD45 ${ }^{\text {neg }}$ cells expressing epithelial markers, independently from the expression of mesenchymal markers; FACS: Fluorescence-activated cell sorting; FDA: Food and Drug Administration; FITC: Fluorescein isothiocyanate; MBC: Metastatic breast cancer; MES: Subset of CD45 ${ }^{\text {neg }}$ expressing mesenchymal markers only; NEG: Subset of CD45 ${ }^{\text {neg }}$ negative to an antibody cocktail (including CD45, EpCam, E-Cadherin, N-Cadherin, CD44, and CD146); OS: Overall survival; PE: Phycoerythrin; PFS: Progression-free survival; RECIST: Response Evaluation Criteria In Solid Tumors; RT-PCR: Reverse transcriptase-PCR.

\section{Competing interests}

The authors declare that they have no competing interests.

\section{Authors' contributions}

MB optimized the CTC enrichment strategy, optimized the cocktail to identify and characterize CTC, analyzed by DEPArray the peripheral blood samples from MBC patients, sorted single viable CTC for downstream analyses, participated in the analyses of single cell-derived mRNAs, and helped to draft the manuscript. LG recruited the patients, created and updated the clinical database, and performed the statistical analysis. FDB participated in the optimization of the CTC enrichment strategy and in the analysis by DEPArray. SM carried out the genetic analyses of single CTC. MS participated in the CTC enrichment step from MBC blood samples. MT participated in FACS analyses in the definition of the antibody cocktail to be used. GS conceived of the study and participated in the analyses of the DEPArray images. BT participated in FACS analyses in the definition of the antibody cocktail to be used. Ml participated in the design of the study and performed the statistical analysis. CAB conceived the study, participated in its design and coordination, and helped to draft the manuscript. CDL participated in the pathological characterization of breast cancer specimens. APB conceived the study, participated in its design and coordination, and wrote the manuscript. FP conceived the study, coordinated the patient recruitment and follow-up, and helped to draft the manuscript. DC conceived the study, participated in its design and coordination, and wrote the manuscript. All authors read and approved the final manuscript.

\section{Acknowledgements}

This study was supported by FIRB Accordi di programma $2011 \mathrm{Pr}$. RBAP11Z4Z9 2012-2014 "FIERCE-Find New Molecular and Cellular Targets against Cancer", FIRB Accordi di programma 2011 pr. RBAP11ETKA_007 "Nanotechnological Approaches for Tumor Theragnostic", AIRC 5 per mille Special program 2011 Pr. 12214 "Application of Advanced Nanotechnology in the Development of Cancer Diagnostics Tools", and Project ERC-7FP SP 2 IDEAS QUIDPROQUO G.A. n. 269051 "Molecular Nanotechnology for Life Science Applications: Quantitative Interactomics for Diagnostics, Proteomics and Quantitative Oncology".

The funders had no role in study design, data collection, and analysis, decision to publish, or preparation of the manuscript. The authors are also thankful to Dr Manuela Banzi and Dr Elena Peruzzi from Silicon Biosystem for the support in DEPArray use.

\section{Author details}

${ }^{1}$ Department of Medical and Biological Sciences, University of Udine, P.le Kolbe 4, 33100 Udine, Italy. ${ }^{2}$ Department of Oncology, University Hospital of Udine, P.le S.Maria della Misericordia 15, 33100 Udine, Italy. ${ }^{3}$ Institute of Pathology, University Hospital of Udine, P.le S.Maria della Misericordia 15, 33100 Udine, Italy.

Received: 13 November 2015 Accepted: 12 February 2016

Published online: 09 March 2016

\section{References}

1. Alix-Panabieres C, Pantel K. Challenges in circulating tumour cell research. Nat Rev Cancer. 2014;14(9):623-31.

2. Plaks V, Koopman CD, Werb Z. Cancer. Circulating tumor cells. Science. 2013;341(6151):1186-8.

3. Baccelli I, Schneeweiss A, Riethdorf S, Stenzinger A, Schillert A, Vogel V, et al. Identification of a population of blood circulating tumor cells from breast cancer patients that initiates metastasis in a xenograft assay. Nat Biotechnol. 2013;31(6):539-44.

4. Zhang L, Ridgway LD, Wetzel MD, Ngo J, Yin W, Kumar D, et al. The identification and characterization of breast cancer CTCs competent for brain metastasis. Sci Transl Med. 2013;5(180):180ra148.

5. Bidard FC, Peeters DJ, Fehm T, Nole F, Gisbert-Criado R, Mavroudis D, et al. Clinical validity of circulating tumour cells in patients with metastatic breast cancer: a pooled analysis of individual patient data. Lancet Oncol. 2014; 15(4):406-14.

6. Cohen SJ, Punt CJ, lannotti N, Saidman BH, Sabbath KD, Gabrail NY, et al. Relationship of circulating tumor cells to tumor response, progression-free survival, and overall survival in patients with metastatic colorectal cancer. J Clin Oncol. 2008;26(19):3213-21.

7. de Bono JS, Scher HI, Montgomery RB, Parker C, Miller MC, Tissing H, et al. Circulating tumor cells predict survival benefit from treatment in metastatic castration-resistant prostate cancer. Clin Cancer Res. 2008;14(19):6302-9.

8. Zhang L, Riethdorf S, Wu G, Wang T, Yang K, Peng G, et al. Meta-analysis of the prognostic value of circulating tumor cells in breast cancer. Clin Cancer Res. 2012;18(20):5701-10.

9. Scher HI, Jia X, de Bono JS, Fleisher M, Pienta KJ, Raghavan D, et al. Circulating tumour cells as prognostic markers in progressive, castration- 
resistant prostate cancer: a reanalysis of IMMC38 trial data. Lancet Oncol. 2009;10(3):233-9.

10. Lianidou ES, Mavroudis D, Georgoulias V. Clinical challenges in the molecular characterization of circulating tumour cells in breast cancer. $\mathrm{Br} J$ Cancer. 2013;108(12):2426-32.

11. Liu S, Cong Y, Wang D, Sun Y, Deng L, Liu Y, et al. Breast cancer stem cells transition between epithelial and mesenchymal states reflective of their normal counterparts. Stem Cell Reports. 2014;2(1):78-91.

12. Yu M, Bardia A, Wittner BS, Stott SL, Smas ME, Ting DT, et al. Circulating breast tumor cells exhibit dynamic changes in epithelial and mesenchymal composition. Science. 2013;339(6119):580-4

13. Alix-Panabieres C, Pantel K. Technologies for detection of circulating tumor cells: facts and vision. Lab Chip. 2014;14(1):57-62.

14. Parkinson DR, Dracopoli N, Petty BG, Compton C, Cristofanilli M, Deisseroth $A$, et al. Considerations in the development of circulating tumor cell technology for clinical use. J Transl Med. 2012;10:138

15. Fabbri F, Carloni S, Zoli W, Ulivi P, Gallerani G, Fici $P$, et al. Detection and recovery of circulating colon cancer cells using a dielectrophoresis-based device: KRAS mutation status in pure CTCs. Cancer Lett. 2013;335(1):225-31.

16. Eisenhauer EA, Therasse P, Bogaerts J, Schwartz LH, Sargent D, Ford R, et al. New response evaluation criteria in solid tumours: revised RECIST guideline (version 1.1). Eur J Cancer. 2009;45(2):228-47.

17. Lakhani SR, International Agency for Research on Cancer, World Health Organization. WHO classification of tumours of the breast. World Health Organization classification of tumours, 4th edition. Lyon: International Agency for Research on Cancer; 2012.

18. Elston CW, Ellis IO. Pathological prognostic factors in breast cancer. I. The value of histological grade in breast cancer: experience from a large study with long-term follow-up. Histopathology. 2002;41(3A):154-61.

19. Schnitt SJ. Classification and prognosis of invasive breast cancer: from morphology to molecular taxonomy. Mod Pathol. 2010;23 Suppl 2:S60-4.

20. Joosse SA, Gorges TM, Pantel K. Biology, detection, and clinical implications of circulating tumor cells. EMBO Mol Med. 2015;7(1):1-11.

21. Riahi R, Gogoi P, Sepehri S, Zhou Y, Handique K, Godsey J, et al. A nove microchannel-based device to capture and analyze circulating tumor cells (CTCS) of breast cancer. Int J Oncol. 2014;44(6):1870-8.

22. Chavez KJ, Garimella SV, Lipkowitz S. Triple negative breast cancer cell lines: one tool in the search for better treatment of triple negative breast cancer. Breast Dis. 2010;32(1-2):35-48.

23. Barriere G, Fici P, Gallerani G, Fabbri F, Zoli W, Rigaud M. Circulating tumor cells and epithelial, mesenchymal and stemness markers: characterization of cell subpopulations. Ann Transl Med. 2014;2(11):109.

24. Pantel K, Alix-Panabieres C. Real-time liquid biopsy in cancer patients: fact or fiction? Cancer Res. 2013;73(21):6384-8.

25. Schork NJ. Personalized medicine: Time for one-person trials. Nature. 2015; 520(7549):609-11.

26. Riethdorf S, Fritsche H, Muller V, Rau T, Schindlbeck C, Rack B, et al. Detection of circulating tumor cells in peripheral blood of patients with metastatic breast cancer: a validation study of the Cell Search system. Clin Cancer Res. 2007;13(3):920-8

27. Rack B, Schindlbeck C, Juckstock J, Andergassen U, Hepp P, Zwingers T, Friedl TW, Lorenz R, Tesch H, Fasching PA, et al. Circulating tumor cells predict survival in early average-to-high risk breast cancer patients. J Natl Cancer Inst. 2014;106(5).

28. Konigsberg R, Obermayr E, Bises G, Pfeiler G, Gneist M, Wrba F, et al. Detection of EpCAM positive and negative circulating tumor cells in metastatic breast cancer patients. Acta Oncol. 2011;50(5):700-10.

29. Mostert B, Kraan J, Bolt-de Vries J, van der Spoel P, Sieuwerts AM, Schutte M, et al. Detection of circulating tumor cells in breast cancer may improve through enrichment with anti-CD146. Breast Cancer Res Treat. 2011;127(1):33-41.

30. de Albuquerque A, Kaul S, Breier G, Krabisch P, Fersis N. Multimarker Analysis of Circulating tumor cells in peripheral blood of metastatic breast cancer patients: a step forward in personalized medicine. Breast Care. 2012;7(1):7-12.

31. Mostert B, Kraan J, Sieuwerts AM, van der Spoel P, Bolt-de Vries J, Prager-van der Smissen WJ, et al. CD49f-based selection of circulating tumor cells (CTCS) improves detection across breast cancer subtypes. Cancer Lett. 2012;319(1):49-55.

32. Manaresi N, Romani A, Medoro G, Altomare L, Leonardi A, Tartagni M, et al. A CMOS chip for individual cell manipulation and detection. leee J Solid-St Circ. 2003;38(12):2297-305.

33. Jones TB. Basic theory of dielectrophoresis and electrorotation. IEEE Eng Med Biol Mag. 2003;22(6):33-42.
34. De Giorgi U, Valero V, Rohren E, Mego M, Doyle GV, Miller MC, et al. Circulating tumor cells and bone metastases as detected by FDG-PET/CT in patients with metastatic breast cancer. Ann Oncol. 2010;21(1):33-9.

35. Li YM, Pan $Y$, Wei $Y$, Cheng $X$, Zhou BP, Tan $M$, et al. Upregulation of CXCR4 is essential for HER2-mediated tumor metastasis. Cancer Cell. 2004;6(5):459-69.

36. Cristofanilli M, Budd GT, Ellis MJ, Stopeck A, Matera J, Miller MC, et al. Circulating tumor cells, disease progression, and survival in metastatic breast cancer. N Engl J Med. 2004;351(8):781-91.

37. Bedard PL, Hansen AR, Ratain MJ, Siu LL. Tumour heterogeneity in the clinic Nature. 2013;501(7467):355-64.

38. Smerage JB, Barlow WE, Hortobagyi GN, Winer EP, Leyland-Jones B, Srkalovic G, et al. Circulating tumor cells and response to chemotherapy in metastatic breast cancer: SWOG S0500. J Clin Oncol. 2014;32(31):3483-9.

\section{Submit your next manuscript to BioMed Central and we will help you at every step:}

- We accept pre-submission inquiries

- Our selector tool helps you to find the most relevant journal

- We provide round the clock customer support

- Convenient online submission

- Thorough peer review

- Inclusion in PubMed and all major indexing services

- Maximum visibility for your research

Submit your manuscript at www.biomedcentral.com/submit
C) BioMed Central 